DRAFT VERSION JUNE 15, 2021

Preprint typeset using $\mathrm{LT}_{\mathrm{E}} \mathrm{X}$ style emulateapj v. 9/08/03

\title{
A LARGE DARK MATTER CORE IN THE FORNAX DWARF SPHEROIDAL GALAXY?
}

\author{
Louis E. Strigari ${ }^{1,2}$ James S. Bullock $^{1}$, Manoj Kaplinghat ${ }^{1}$, Andrey V. Kravtsov ${ }^{3}$, Oleg Y. Gnedin ${ }^{4}$, \\ Kevork AbaZAJian ${ }^{5}$, Anatoly A. KLyPin ${ }^{6}$ \\ Draft version June 15, 2021
}

\begin{abstract}
We use measurements of the stellar velocity dispersion profile of the Fornax dwarf spheroidal galaxy to derive constraints on its dark matter distribution. Though the data are unable to distinguish between models with small cores and those with cusps, we show that a large $\gtrsim 1 \mathrm{kpc}$ dark matter core in Fornax is highly implausible. Irrespective of the origin of the core, reasonable dynamical limits on the mass of the Fornax halo constrain its core radius to be no larger than $\sim 700 \mathrm{pc}$. We derive an upper limit of $r_{\text {core }} \lesssim 300 \mathrm{pc}$ by demanding that the central phase-space density of Fornax not exceed that directly inferred from the rotation curves of low-mass spiral galaxies. Further, if the halo is composed of warm dark matter then phase-space constraints force the core to be quite small in order to avoid conservative limits from the Ly $\alpha$ forest power spectrum, $r_{\text {core }} \lesssim 85$ pc. We discuss our results in the context of the idea that the extended globular cluster distribution in Fornax can be explained by the presence of a large $\sim 1.5 \mathrm{kpc}$ core. A self-consistent core of this size would be drastically inconsistent with the expectations of standard warm or cold dark matter models, and would also require an unreasonably massive dark matter halo, with $V_{\max } \simeq 200 \mathrm{~km} \mathrm{~s}^{-1}$.

Subject headings: cosmology: dark matter, cosmology: observations, cosmology: theory, galaxies: kinematics and dynamics, galaxies: structure, galaxies: formation, dwarfs: galaxies
\end{abstract}

\section{INTRODUCTION}

The central density distribution in many dark matter dominated galaxies appears to be lower than what is expected in standard $\Lambda+$ Cold Dark Matter $(\Lambda C D M)$ models (Moore 1994; Flores \& Primack 1994; de Blok et al. 2001; Zentner \& Bullock 2002; Kuhlen et al. 2005; Simon et al. 2005; Zackrisson et al. 2006). This discrepancy, along with other potential difficulties (e.g. Klypin et al. 1999; Moore et al. 1999), can be ameliorated by considering alternative dark matter models (e.g. Zentner \& Bullock 2003, and references therein). One particularly intriguing scenario is Warm Dark Matter (WDM), which differs from CDM in that the dark matter has a nonnegligible velocity dispersion (Bond et al. 1980; Blumenthal et al. 1982; Pagels \& Primack 1982; Dodelson \& Widrow 1994; Hogan \& Dalcanton 2000; Abazajian 2006a). Generally, these WDM models come with two distinct observational signatures. First, there is a reduction in clustering and a delay in collapse times for structures on linear scales approximately smaller than the free-streaming scale of the WDM particle. This effect is currently probed by measurements of the Ly $\alpha$ forest power spectrum and this places strong constraints on the mass of the WDM particle (Viel et al. 2005; Abazajian 2006b; Seljak et al. 2006). Second, the velocity dispersion of WDM imposes an upper limit on the phase-space density, defined as $Q \equiv \rho / \sigma^{3}$, where $\rho$ is the density and $\sigma$ is the velocity dispersion of the dark matter. The upper limit on $\mathrm{Q}$ implies that the density profiles of WDM halos must saturate to form a constant density core at an inner radius defined by $Q<Q_{\max }$ (Tremaine \& Gunn 1979). This is in stark con-

\footnotetext{
1 Center for Cosmology, Dept. of Physics \& Astronomy, University of California, Irvine, CA; 1strigar@uci.edu

${ }^{2}$ McCue Fellow

${ }^{3}$ Dept. of Astronomy \& Astrophysics, KICP, Enrico Fermi Institute, 5640 S. Ellis Ave., The University of Chicago, Chicago, IL 60637

${ }^{4}$ Astronomy Department, The Ohio State University, Columbus, $\mathrm{OH}$

5 Theoretical Division, MS B285, Los Alamos National Laboratory, Los Alamos, NM 87545

${ }^{6}$ Astronomy Dept, New Mexico State University, Las Cruces, NM 88001
}

trast to CDM predictions that give dark halo density profiles that rise steeply towards the center, $\rho \propto r^{\gamma}$, with $\gamma \sim 1$, (e.g. Dubinski \& Carlberg 1991; Navarro et al. 1996; Moore et al. 1999; Klypin et al. 2001; Navarro et al. 2004; Diemand et al. 2005) and have divergent phase-space profiles $Q \propto r^{-1.9}$ (Taylor \& Navarro 2001).

Dwarf spheroidal galaxies (dSph's) are diffuse, lowluminosity systems, with a total mass believed to be dominated by their host dark matter halos (Mateo 1998). Assuming that these galaxies are in dynamical equilibrium, the stars act as tracers of the gravitational potential, and can be used as a probe of the dark matter density profile. Indeed, dSph's provide a unique testing ground for the nature of dark matter, because in these low-mass systems the phase-space cores are the most observationally accessible (Sellwood 2000; Dalcanton \& Hogan 2001; Alam, Bullock, \& Weinberg 2002). Unfortunately, attempts to constrain uniquely the dark matter profiles of dwarfs using stellar velocity dispersion data are plagued by degeneracies. We emphasize here that while the data are currently unable to settle the question of a central density core versus cusp, they do provide powerful constraints on the combination of core size $\left(r_{\text {core }}\right)$ and halo maximum circular velocity $\left(V_{\max }\right)$. Each solution maps directly to a constraint on $Q$.

The Fornax dSph is approximately $138 \mathrm{kpc}$ from the Milky Way (e.g., Buonanno et al. 1999), in the vicinity of its orbital pericenter (Dinescu et al. 2004). Its stellar population is dominated by intermediate age stars $(\sim 4 \mathrm{Gyr})$, although Fornax has also both very young stars $(\sim 0.2-2$ Gyr $)$ and very old stars ( $\gtrsim 10$ Gyr, e.g., Pont et al. 2004, and references therein). Among the most puzzling observed properties of Fornax is the fact that it hosts 5 globular clusters distributed over a wide range of distances from the center of this galaxy. Standard arguments suggest that (in the absence of external heating) these systems should have sunk to the center via dynamical friction in much less than a Hubble time (Oh et al. 2000). Recently, Goerdt et al. (2006, G06 hereafter) and Sanchez-Salcedo et al. (2006) suggested that the presence of these clusters provides 

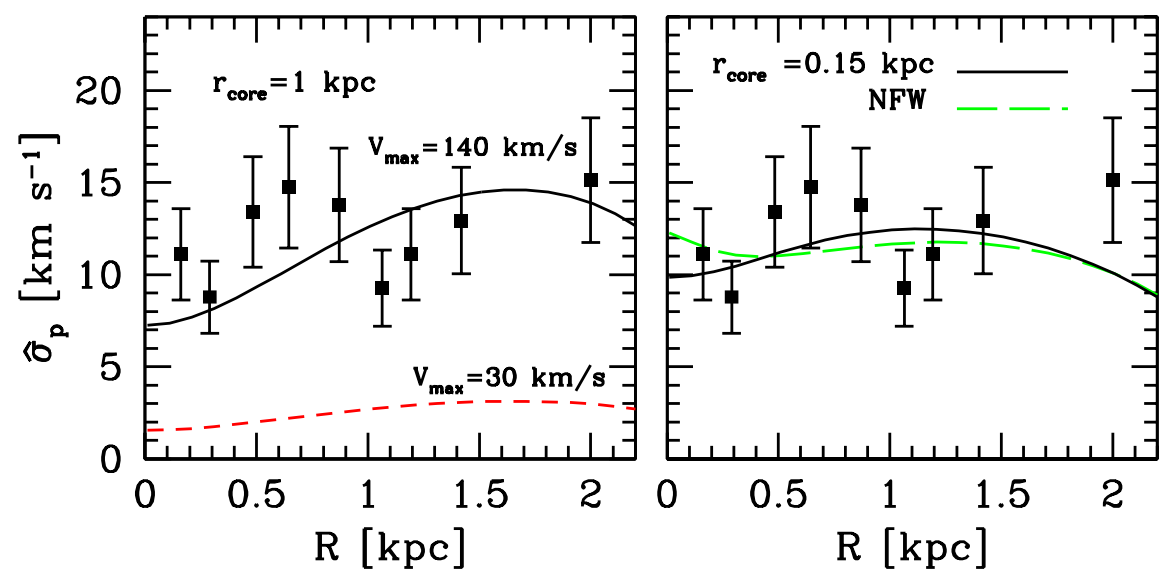

FIG. 1. - Measurements of the Fornax velocity dispersion profile compared to models for the dark matter halo. Left panel: Predictions for the case of a fixed $r_{\text {core }}=1 \mathrm{kpc}$; the solid curve shows a large $V_{\max }=140 \mathrm{~km} / \mathrm{s}$, and the dashed curve shows a small $V_{\max }=30 \mathrm{~km} / \mathrm{s}$. Right panel: The best-fitting cases for two distinct density profiles: the solid curve shows the fit for the $\alpha=1.5$ profile described in the text with $V_{\max }=28 \mathrm{~km} / \mathrm{s}$, and the long-dashed curve shows the best-fitting cuspy NFW profile with $V_{\max }=50 \mathrm{~km} / \mathrm{s}$.

good evidence for the existence of a large, constant density dark matter core in the center of Fornax. Specifically, G06 argued that the globular clusters will stall in their orbital decay if they encounter a constant density core. Thus the globular clusters should be located in a narrow range of radii from the center of Fornax corresponding to its halo core size. The Fornax globular clusters are observed to orbit at a range of projected distances from the center of galaxy of between 0.24 and $1.60 \mathrm{kpc}$ (Mackey \& Gilmore 2003). This suggests that a core size $r_{\text {core }} \gtrsim 1.5 \mathrm{kpc}$ would be required to explain the observed distribution. Another idea, which relies on a somewhat finetuned timing argument, is that only the central-most globular cluster is stalled at the core, $r_{\text {core }} \gtrsim 240 \mathrm{pc},(\mathrm{G} 06$; J. Read, private communication) and the other four globular clusters were simply formed at a large enough radius that they have not sunk to the center.

In this paper we consider the general plausibility of a large core in Fornax. In section 2, we focus on the allowed dark halo solutions which reproduce the observed stellar kinematics, and we present a toy model to understand the nature of the these solutions. In section 3, we combine these solutions with limits on the WDM mass from the Ly $\alpha$ forest power spectrum to place constraints on the maximal core size. We also discuss additional, more independent constraints on the core size. We conclude by discussing these results in the context of the observed globular cluster distribution in Fornax.

\section{DYNAMICAL CONSTRAINTS ON THE DARK HALO OF FORNAX}

For a stellar system in equilibrium and embedded in a spherically symmetric dark matter potential, the radial stellar velocity dispersion $\sigma_{r}(r)$ is given by the Jeans equation:

$$
r \frac{d\left(\rho_{\star} \sigma_{r}^{2}\right)}{d r}=-\rho_{\star}(r) V_{c}^{2}(r)-2 \beta(r) \rho_{\star} \sigma_{r}^{2} .
$$

Here the circular velocity is governed by the mass distribution $V_{\mathrm{c}}^{2}(r)=G M(r) / r$ and the anisotropy parameter is $\beta \equiv$ $1-\sigma_{\theta}^{2} / \sigma_{r}^{2}$, where $\sigma_{r}$ and $\sigma_{\theta}$ are the radial and angular velocity dispersions, respectively. We consider $\beta(r)$ to be constant in radius, and explore three choices of $\beta=-0.5,0$, and 0.5 , using $\beta=0$ as our fiducial case. The appropriate value for $\beta$ is unknown, however this range brackets reasonable choices. Dissipationless CDM simulations show that $\beta$ for dark matter increases from $\sim 0$ to 0.6 as the radius increases towards the virial radius of the halo (Cole \& Lacey 1996; Colín et al. 2000; Diemand et al. 2004; Faltenbacher et al. 2005; Wojtak et al. 2005). However, models of dSph galaxies (e.g. Mayer \& Wadsley 2004) suggest that a more natural value for the stars may be negative, $\beta \sim-0.5$. Our main results are not sensitive to the choice of $\beta>0$, and we note that $\beta<0$ provides an even stronger upper limit on the core size than the $\beta=0$ case.

We assume that the stellar component and the dark matter component are uncoupled, each with an independent density distribution, and that $M(r)$ is dominated by the dark matter. We model the stellar distribution, $\rho_{*}(r)$, as a spherically symmetric King profile (King 1962) and adopt $r_{\mathrm{c}}=0.39 \mathrm{kpc}$ and $r_{\mathrm{t}}=2.7 \mathrm{kpc}$ for its King core and tidal radii (Mateo 1998). For the dark matter, we consider a density profile of the form

$$
\rho(r)=\frac{\rho_{0}}{\left[1+\left(r / r_{0}\right)^{\alpha}\right]^{3 / \alpha}},
$$

where $\rho_{0}$ is a central core density and $r_{0}$ is a characteristic radius. Note that we demand that the profile falls off at large radius as $r^{-3}$, as seen in isolated galaxies (Prada et al. 2003). The parameter $\alpha$ controls the sharpness of the transition from flat to falling density with radius. In our fiducial case we adopt $\alpha=1.5$. This choice yields a profile that matches very closely that advocated by Burkert (1995) to match rotation curve data for low-mass galaxies. However, our conclusions do not change considerably if we adopt other reasonable choices $\alpha=1.0$ or 2.0 (see below). Note that once $\alpha$ is fixed, any two independent parameters in the mass distribution (e.g. $r_{0}$ and the maximum of the circular velocity, $V_{\max }$ ) define the density profile completely. The circular velocity curve implied by equation 2 peaks at a radius $r_{\max }=(4.4,3.2,2.9) r_{0}$ for $\alpha=(1.0,1.5,2.0)$.

The log-slope of this profile, $\gamma$, gradually approaches zero towards the center, but a constant density "core radius" is never achieved at finite $r$. A natural way to define a core radius 


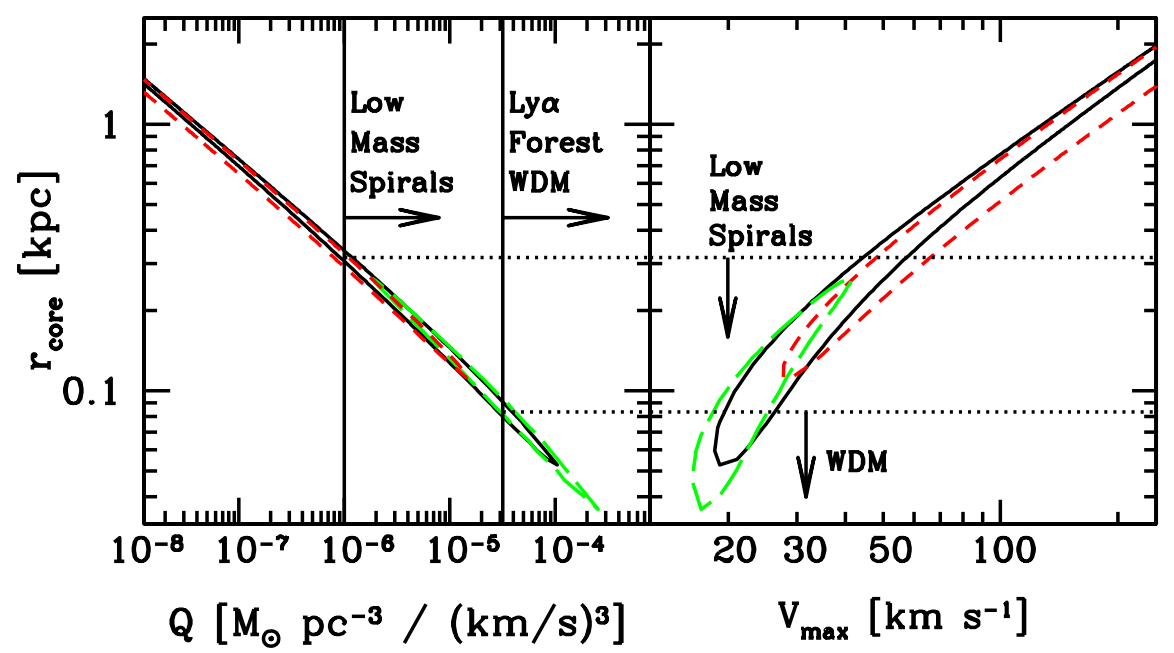

FIG. 2.- Constraints on the core radius of Fornax as a function of central phase-space density (left) and maximum circular velocity (right) derived from the velocity dispersion profile. We define $r_{\text {core }}$ as the radius where the log-slope of the density profile is $\gamma=-0.1$ in equation 2 with $\alpha=1.5, r_{\text {core }} \simeq 0.1 r_{0}$. The long-dashed, solid, and short-dashed lines use $\beta=0.5,0.0$, and -0.5 respectively. Values of $r_{\text {core }}$ with $Q \gtrsim 3 \times 10^{-5} \mathrm{M}_{\odot} \mathrm{pc}^{-3}(\mathrm{~km} / \mathrm{s})^{-3}$ are ruled out by Ly $\alpha$ forest constraints in the case of WDM with $\mathrm{g}=2\left(r_{\text {core }} \lesssim 85 \mathrm{pc}\right)$. In a more general class of dark matter models, directly observed phase-space limits from low-mass spiral rotation curves, $Q \gtrsim 10^{-6} \mathrm{M}_{\odot} \mathrm{pc}^{-3}(\mathrm{~km} / \mathrm{s})^{-3}$, demand $r_{\text {core }} \lesssim 300 \mathrm{pc}$. Most generally and irrespective of the cause of the core, a Fornax halo with $V_{\max } \gtrsim 100 \mathrm{~km} \mathrm{~s}^{-1}$ is disfavored from dynamical considerations. This implies $r_{\text {core }} \lesssim 700 \mathrm{pc}$.

in this case is to use the radius where $\gamma$ reaches some small value, such that $r_{\text {core }} \equiv r_{\gamma}$, where $r_{\gamma}$ is defined as follows:

$$
r_{\gamma}=r_{0}[-3 / \gamma-1]^{-1 / \alpha} .
$$

More general forms for $\rho(r)$ may be considered, but this logslope definition of core radius is always possible. In this paper we define the core radius to be where $\gamma=-0.1$. This is the same definition used by G06. For $\alpha=1.5$ we have $r_{\text {core }} \simeq$ $0.1 r_{0}$.

In order to compare to measurements of the radial stellar velocity dispersion profile, we project equation 1 along the line-of-sight to the observer. Performing this projection gives

$$
\hat{\sigma}_{p}^{2}(R)=\frac{2}{I(R)} \int_{R}^{\infty}\left(1-\beta \frac{R^{2}}{r^{2}}\right) \frac{\rho_{\star} \sigma_{r}^{2} r}{\sqrt{r^{2}-R^{2}}} d r,
$$

where $I(R)$ is the surface density as determined from $\rho_{\star}$, and $R$ is the radial distance from the center of the galaxy. The lineof-sight stellar velocity dispersion was recently measured by Walker et al. (2005). The authors utilize a primary sample of $N=209$ stars and perform cuts to remove interloping stars, and present data for three removal schemes that yield $N=179$, $N=185$, and $N=189$ stars respectively. For our analysis, we use their $N=185$ sample, as shown by the points with with error bars in Figure 1. Our conclusions are not strongly affected by this particular choice.

The lines in Figure 1 show the predicted $\hat{\sigma}_{p}$ profiles for four different input dark matter density distributions compared to the data. In each case we have assumed $\beta=0$. Each curve in the left panel assumes $\alpha=1.5$ in equation 2 and fixes the core size to $r_{\text {core }}=1 \mathrm{kpc}$. The solid line shows the best-fit case to the data with these restrictions and has $V_{\max }=140 \mathrm{~km} \mathrm{~s}^{-1}$. The dashed line, on the other hand, corresponds to what might be considered a more reasonable halo mass, with $V_{\max }=30 \mathrm{~km} \mathrm{~s}^{-1}$. Clearly, in order to reproduce a reasonable stellar velocity dispersion profile with such a large core, the dark matter halo of Fornax must be quite massive. In order to match the data with $V_{\max }=30 \mathrm{~km} \mathrm{~s}^{-1}$, a smaller core must be used. The solid line in the right panel uses the same profile shape with $V_{\max }=30 \mathrm{~km} \mathrm{~s}^{-1}$, but now with $r_{\text {core }}=0.15 \mathrm{kpc}$. There is a clear degeneracy between $V_{\max }$ and $r_{\text {core }}-$ a large flat density distribution requires a deeper potential well in order to reproduce the data. The dashed line in the right hand panel shows a best-fit case for a (cuspy) NFW dark matter profile. Note that these data do not exclude an inner cusp.

Figure 2 illustrates a more general exploration of the allowed parameter space for $\alpha=1.5$. We demand that the allowed fit region obey $\chi^{2}<\chi_{\min }^{2}+\Delta \chi^{2}$, where $\Delta \chi^{2}=4.6$ defines the $90 \%$ confidence level for a $\chi^{2}$ distribution with two degrees of freedom. The parameters $r_{\text {core }}$ and $V_{\max }$ define the density profile and must lie within the banana-shaped contour in the right panel in order to reproduce the observed $\hat{\sigma}_{p}$. At large $V_{\max }$ the contours follow $r_{\text {core }} \propto V_{\max }$ and we discuss how this can be understood analytically below. The left panel shows the same best-fit parameter space, now expressed in terms of $r_{\text {core }}$ and the central $(r=0)$ phase-space density of the dark matter, $Q$, implied by each solution. The different line types in Figure 2 correspond to different assumed values for the velocity anisotropy: $\beta=-0.5$ (short-dash), $\beta=0$ (solid), and $\beta=0.5$ (long-dash). Note that for $\beta=0.5$ solutions with $r_{\text {core }} \gtrsim 250 \mathrm{pc}$ and $V_{\max } \gtrsim 30 \mathrm{~km} \mathrm{~s}^{-1}$ are disfavored. For $\beta=-0.5$ solutions with $V_{\max } \lesssim 30 \mathrm{~km} \mathrm{~s}^{-1}$ are disfavored.

Similar results for the velocity anisotropy for Fornax were uncovered in previous studies (tokas 2002; Kazantzidis et al. 2004). In particular Łokas (2002) showed that the degeneracy with $\beta$ and the density profile can be broken by considering higher order moments of the velocity distribution function. Though not shown, we have also explored the effects of varying the specific dark halo profile shape by varying $\alpha$ in equation 2. As $\alpha$ increases, the allowed core size increases at fixed $V_{\max }$. For example, at $V_{\max }=30 \mathrm{~km} \mathrm{~s}^{-1}$ the best-fit value of $r_{\text {core }}$ varies from $50 \mathrm{pc}$ to $250 \mathrm{pc}$ as $\alpha$ varies from 1.0 to 2.0. We discuss the origin of this variation in the next section.

Note that while some large values of $r_{\text {core }}(\sim \mathrm{kpc})$ are al- 
lowed by the velocity dispersion data, they require very large values of $V_{\max }\left(\gtrsim 100 \mathrm{~km} \mathrm{~s}^{-1}\right)$. One may be concerned that the quoted $V_{\max }$ values represent an unphysical extrapolation to large $r_{\max }$ radii based on constraints derived by stellar velocity dispersions at $\sim 2 \mathrm{kpc}$. However, direct observational evidence suggests that this is a reasonable extrapolation. Not only is our adopted profile shape motivated by rotation curve fits (Burkert 1995) but large dark matter halos around isolated galaxies are known to decline no faster than $r^{-3}$ at large $r$ (Prada et al. 2003). In this case, the only effect that could act to truncate this fall-off is tidal striping. If Fornax is at a distance $D$ from the Milky Way, then the tidal radius may be estimated via the Jacobi approximation $R_{t} \simeq D\left(M_{\text {Fornax }}\left(<R_{t}\right) /\left(3 M_{\mathrm{MW}}(<D)\right)\right)^{1 / 3}$. For a flat, Milky Way rotation curve with $V_{\max }=220 \mathrm{~km} \mathrm{~s}^{-1}$ we obtain $R_{t} \simeq 80 \mathrm{kpc}\left(V_{\max } /\left(200 \mathrm{~km} \mathrm{~s}^{-1}\right)\right)^{2 / 3}$. Compare this to our central $V_{\max } \simeq 200 \mathrm{~km} \mathrm{~s}^{-1}$ solution, which gives $r_{\max }=3.20=$ $32 r_{\text {core }} \simeq 48 \mathrm{kpc}$ and we see that the peak rotation curve should be little affected by tidal truncation. We conclude that while a large Fornax halo may be implausible on physical grounds (see below) the derived constraint itself is meaningful.

It is also possible that the large $V_{\max }$ end of the contour is affected by the presence of interloping stars, and that the contours actually close at a smaller value of $V_{\max }$ (and $r_{\text {core }}$ ). In particular, removal of interlopers is most important in the outer regions of the galaxy. To examine the effect of interlopers, we have performed an independent fit of the data presented in Walker et al. (2005). We use the same number of radial bins, and in each bin we assume that the distribution of stars in velocity follows a Gaussian plus a constant, where the constant accounts for interlopers. The main difference we find is that there is a lower dispersion in the last bin at $\sim 2$ kpc than in Walker et al. (2005), with a larger error in this bin. These results do not change the shape of the constraints at low $V_{\max }$, but disfavor large values: $V_{\max } \gtrsim 65 \mathrm{~km} \mathrm{~s}^{-1}$ and $r_{\text {core }} \gtrsim 400 \mathrm{pc}$ are excluded.

While uncertainties associated with interlopers stars may affect the closure of the contour in Figure 2 at large values of $V_{\max }$, the lower part of the contour is more robust to these uncertainties. Indeed, it is this region of the diagram, and the $V_{\max }-r_{\text {core }}$ degeneracy itself which allows us to use the implied phase-space densities in these galaxies to limit core sizes. Before going on to discuss these limits, we discuss the origin of this degeneracy using analytic arguments and extend these arguments to explore the dependency of our constraints on the assumed underlying dark profile shape. Readers interested in our resulting constraints on the core size of Fornax may skip ahead to section 3 .

\subsection{Toy Model to Understand the Solutions from Stellar Kinematics}

Here we show that the scaling of the contours in the $V_{\max }$ $r_{\text {core }}$ can be understood in the context of a toy model. For simplicity we will consider $\beta=0$. From equation 1 we see that if the stellar mass is sub-dominant, the stellar velocity dispersion is governed by the circular velocity curve of the dark matter $V_{c}(r)$ within the stellar radius, $r_{\star} \simeq r_{c} \simeq 0.5 \mathrm{kpc}$. Here $r_{\star}$ corresponds to a weighted average of the stellar material over an integral $\int \mathrm{d} r \rho_{\star}(r) V_{c}^{2}(r) / r$. Thus $r_{\star}$ will typically be smaller than the half-mass radius of the stars.

Consider now a toy-model dark matter halo with a rotation curve given by

$$
V_{c}(r)= \begin{cases}a V_{\max }\left(\frac{r}{r_{\text {core }}}\right) & r<r_{\text {core }} \\ a V_{\max }\left(\frac{r}{r_{\text {core }}}\right)^{1 / 2} & r_{\text {core }}<r<r_{\text {flat }} \\ V_{\max } & r>r_{\text {flat }}=r_{\text {core }} / a^{2} .\end{cases}
$$

This corresponds to a density profile with a constant density at $r<r_{\text {core. }}$. The profile transitions to an $\rho \propto r^{-1}$ regime beyond the core and finally reaches an isothermal distribution, $\rho \propto r^{-2}$, at $r>r_{\text {flat }}$. We assume that the transitions occur in a small region around $r_{\text {core }}$ and $r_{\text {flat }}$ and that the density profile is continuous. The variable $a \leq 1$ parameterizes the sharpness of the transition from a core to flat rotation, and can be viewed as analogous to the parameter $\alpha$ in equation 2. For $a=1$, the transition is sudden, $r_{\text {core }}=r_{\text {flat }}$, while for $a \ll 1$ the rotation curve rises very gradually and reaches $V_{\max }$ at $r=r_{\text {flat }}=r_{\text {core }} / a^{2}$. Note that the value of the rotation velocity at $r=r_{\text {core }}$ is $V_{\text {core }}=a V_{\max }$, and thus decreases at fixed $V_{\max }$ as the transition is made more gradual. Also note that the density in the core of the halo $\rho_{0}$ is proportional to $a V_{\max } / r_{\text {core }}$.

Now consider a stellar distribution with radius $r_{\star}$ and with constant density, $\rho_{*}(r)=$ constant, embedded within the gravitationally-dominant dark halo. If the halo has a very sharp transition from the core region to flat rotation, $a=1$ and $r_{\text {core }}=r_{\text {flat }}$, then equation 1 implies that the central stellar radial velocity dispersion, $\sigma_{\star}^{0}=\sigma_{\star}(r=0)$, is

$$
\sigma_{\star}^{0}= \begin{cases}V_{\max } \frac{1}{\sqrt{2}}\left(\frac{r_{\star}}{r_{\text {core }}}\right) & r_{\star} \leq r_{\text {core }} \\ V_{\max }\left[\frac{1}{2}+\ln \left(r_{\star} / r_{\text {core }}\right)\right]^{1 / 2} r_{\star}>r_{\text {core }}=r_{\text {flat }} .\end{cases}
$$

This suggests that if we fix the central velocity dispersion, the maximum circular velocity of the halo is degenerate with the core radius, and must increase with core radius as $V_{\max } \propto r_{\text {core }}$ in the large-core regime. Another way to interpret the result is that in this regime, the core density of the dark matter halo is fixed, but not the core size. Conversely, in the small core regime we expect $V_{\max }$ to vary slowly with increasing core size $\left(V_{\max } \propto \sqrt{1+2 \ln \left(r_{\star} / r_{\text {core }}\right)}\right.$ for this example). Interestingly, these qualitative features are demonstrated by our bestfit contours in the right panel of Figure 2.

For a more gradual transition from core to flat rotation, we allow $a<1$ with $r_{\text {core }}<r_{\text {flat }}$. In this case, for $r_{\star}<r_{\text {flat }}$, we have

$$
\sigma_{\star}^{0}=\left\{\begin{array}{l}
a V_{\max } \frac{1}{\sqrt{2}}\left(\frac{r_{\star}}{r_{\text {core }}}\right) \quad r_{\star} \leq r_{\text {core }} \\
a V_{\max }\left[\frac{r_{\star}}{r_{\text {core }}}-\frac{1}{2}\right]^{1 / 2} r_{\star}>r_{\text {core }} .
\end{array}\right.
$$

This means that for a fixed core size, a weaker transition $(\operatorname{smaller} a)$ requires a larger $V_{\max }$ to reproduce a given $\sigma_{*}^{0}$ : $V_{\max } \propto r_{\text {core }} / a$ or fixed halo core density in the large-core regime, and $V_{\max } \propto \sqrt{r_{\text {core }}} / a$ in the small-core regime $\left(r_{\text {core }} \ll\right.$ $\left.r_{\star}\right)$. This result demonstrates qualitatively why the implied core sizes increase with a sharper transition in the assumed dark matter profile ( $\alpha: 1 \rightarrow 2$ in equation 2$)$. The stellar velocity dispersion is roughly governed by $V_{c}\left(r \simeq r_{\star}\right)$. As the transition becomes sharper, the ratio $V_{c}\left(r=r_{\star}\right) / V_{\max }$ increases, and an observed stellar velocity dispersion can be produced with a smaller $V_{\max }$.

The scalings seen in the left-hand panel of Figure 2 for $Q=$ $\rho_{0} / \sigma_{\mathrm{dm}}^{3}$ vs. $V_{\max }$ can be similarly understood. In the largecore regime, where $r_{\star} \lesssim r_{\text {core }}$, the central density $\rho_{0}$ will be approximately constant because fixing $\sigma_{\star}^{0}$ roughly fixes $V_{c}\left(r_{\star}\right)$. 
In this case, we expect $Q \propto \sigma_{\mathrm{dm}}^{-3} \propto\left(a V_{\max }\right)^{-3} \propto r_{\text {core }}^{-3}$, which reproduces the contour scaling in Figure 2.

The dependence of $Q$ on the transition parameter $a$ is weaker than the scaling of $V_{\max }: Q^{-1} \propto a V_{\max } r_{\text {core }}^{2}[1-$ $2 \ln (a)]^{3 / 2}$. This result is consistent with our numerical findings: the sharper the transition, the larger is the core size required for a given $Q$. In the small core regime, we have seen that $a V_{\max } \propto \sqrt{r_{\text {core }}}$ and hence $Q \propto r_{\text {core }}^{-2.5}$ implying that the $r_{\text {core }}$ vs $Q$ contour should steepen slightly for small values of $r_{\text {core }}$.

\section{RESULTS}

\subsection{Phase-Space Constraints}

The derived relationship between allowed $V_{\max }$ and $r_{\text {core }}$ values becomes interesting when expressed in terms of the implied central phase-space density for each solution. Consider the case of WDM with a distribution function given by

$$
f=g \frac{\beta}{e^{p / T}+1},
$$

where $g$ is the number of spin degrees of freedom of the particle. For $\beta=1$ the distribution is thermal, and in this case we define the mass of the WDM particle as $m_{x}$. An example of a (non-thermal) $\beta \neq 1$ distribution is WDM from an oscillationproduced sterile neutrino (e.g. Dodelson \& Widrow 1994). If $\beta$ is independent of momentum, we may calculate the maximum phase-space density by fixing the present-day density of dark matter and calculating the velocity dispersion from equation 8 :

$$
Q_{\max }=5 \times 10^{-4} \beta\left(\frac{g}{2}\right)\left(\frac{m_{x}}{1 \mathrm{keV}}\right)^{4} \mathrm{M}_{\odot} \mathrm{pc}^{-3}\left(\mathrm{~km} \mathrm{~s}^{-1}\right)^{-3} .
$$

As discussed in the introduction, the (course-grained) phasespace density in any WDM galaxy halo can never exceed $Q_{\max }$.

Measurements of the Ly $\alpha$ power spectrum place a limit on the free streaming scale (or equivalently, the present-day velocity) of WDM particles. We can convert this directly to a lower limit on $Q$. For a fixed free-streaming scale, the mass of a thermal and non-thermal particle is related by $m_{x} / T_{x}=m_{s} / T_{s}$, where $m_{s}$ is the mass of the non-thermal particle and $T_{s}$ is its corresponding temperature in equation 8 . Fixing the present-day density of dark matter in this case implies $\beta=\left(m_{s} / m_{x}\right)^{4}$ as long as $\beta$ is independent of momentum. Note that since $\beta$ and $Q$ both scale as the fourth power of the WDM particle mass, any Ly $\alpha$ forest constraint can be mapped uniquely to a $Q_{\max }$ constraint, independent of the particle mass.

The most conservative quoted constraint from the $\operatorname{Ly} \alpha$ forest power spectrum expressed as a limit on the thermal WDM particle mass is $m_{x} \gtrsim 0.5 \mathrm{keV}$ (Viel et al. 2005; Abazajian 2006b). A distinct analysis using different simulations has derived an even stronger limit $m_{x} \gtrsim 2.5 \mathrm{keV}$ (Seljak et al. 2006). The $m_{x} \gtrsim 0.5 \mathrm{keV}$ limit implies an upper limit on the phase-space density in the core of any galaxy of $Q \gtrsim$ $3 \times 10^{-5} \mathrm{M}_{\odot} \mathrm{pc}^{-3}\left(\mathrm{~km} \mathrm{~s}^{-1}\right)^{-3}$. That is, if WDM accounts for the mass of the dark halo of Fornax, then Figure 2 implies that the core must be quite small, $r_{\text {core }} \lesssim 85 \mathrm{pc}$, and $V_{\max } \lesssim 35 \mathrm{~km} \mathrm{~s}^{-1}$ (the lower dotted line). The more stringent $m_{x} \gtrsim 2.5 \mathrm{keV}$ constraint implies $Q \gtrsim 0.02 \mathrm{M}_{\odot} \mathrm{pc}^{-3}\left(\mathrm{~km} \mathrm{~s}^{-1}\right)^{-3}$ and demands $r_{\text {core }} \ll 10 \mathrm{pc}$ even with allowances for extremely sharp transitions to the profile core.

Of course, the Ly $\alpha$ forest constraint only applies to the specific case of a WDM particle with a phase-space density given by equation 9 . More generally, there are models with a finite primordial phase-space density that do not have the same mapping between $Q$ and $P(k)$ as does WDM. For example, the well-motivated SuperWIMP scenario suggests that dark matter arises from out-of-equilibrium decays and this gives rise to a non-thermal spectrum (Cembranos et al. 2005; Kaplinghat 2005). Also, non-thermal resonantly-produced sterile neutrino dark matter would be "cooler" than a thermal or nonresonant sterile WDM candidate (Shi \& Fuller 1999; Abazajian et al. 2001). Yet another example is fuzzy dark matter (Hu et al. 2000).

In cases such as these the relation between the phase-space density and the power spectrum is different from that implied by equation 9 , a more direct observational constraint on $Q$ may be derived from considering the rotation curves of low-mass spiral galaxies (Dalcanton \& Hogan 2001; Kormendy \& Freeman 2004). Among the highest-resolution rotation curves for low-mass spirals are those presented by Simon et al. (2005), who used two-dimensional $\mathrm{H} \alpha$ velocity fields to extract rotation curves for five galaxies. When the dark matter halo components of these galaxies are fit to cored density distributions the central densities range from $\rho_{0} \simeq 0.1-0.5 \mathrm{M}_{\odot} \mathrm{pc}^{-3}$ and the rotation curves flatten at $V_{\max } \gtrsim 74-114 \mathrm{~km} \mathrm{~s}^{-1}$. If we adopt $\sigma_{\mathrm{dm}} \simeq 0.55 V_{\max }$ (as is appropriate for the cored profiles we have used here) then the implied central phase-space limits are $Q \gtrsim(1.7,4.4,1.7,1.6$, and 0.5) $\times 10^{-6} \mathrm{M}_{\odot} \mathrm{pc}^{-3}\left(\mathrm{~km} \mathrm{~s}^{-1}\right)^{-3}$ for NGC2976, NGC4605, NGC5949, NGC5963, and NGC6689, respectively. If we (conservatively) adopt $Q \gtrsim 10^{-6} \mathrm{M}_{\odot} \mathrm{pc}^{-3}\left(\mathrm{~km} \mathrm{~s}^{-1}\right)^{-3}$ as a (direct) constraint on $Q$, this provides a fairly clean upper-limit on the Fornax core radius, $r_{\text {core }} \lesssim 300 \mathrm{pc}$, for a broad class of dark matter candidates (upper dotted line in Figure 2).

\subsection{Phase-Space Independent Constraints}

While the most popular alternatives to CDM produce cores because of their phase-space constraints, cores in dark matter halos need not arise as a result of large velocity dispersions. For example, self-interaction or self-annihilation would produce cores even if the dark matter is cold (Spergel \& Steinhardt 2000; Medvedev 2000; Kaplinghat et al. 2000; SánchezSalcedo 2003). Astrophysical or dynamical effects could also produce cored profiles. For our most general constraint we can use the $r_{\text {core }}-V_{\max }$ relation demanded by the velocity dispersion data to place a reasonable upper limit on the core size in Fornax by imposing a plausible upper limit on $V_{\max }$ for its halo.

Models of Fornax with large $V_{\max }$ have large masses. As a result, they will experience large dynamical friction and spiral to the galactic center very fast. Therefore only in the rare circumstance of a very recent accretion event could a massive subhalo at a distance of $\sim 140 \mathrm{kpc}$ from the Milky Way be possible. Zentner \& Bullock (2003) found that only $5 \%$ of Milky Way-sized $\Lambda$ CDM halos contain a subhalo with $V_{\max }>100 \mathrm{~km} \mathrm{~s}^{-1}$ and that the fraction falls sharply beyond this point. Since the presence of dark matter cores would only decrease the subhalo population, we may adopt $V_{\max } \lesssim$ $100 \mathrm{~km} \mathrm{~s}^{-1}$ as a reasonable upper limit on the Fornax halo. This gives $r_{\text {core }} \lesssim 700 \mathrm{pc}$ as a conservative general upper limit on the core size of Fornax.

In light of this argument, a core large enough to explain the extended distribution of globular clusters in Fornax, $r_{\text {core }} \simeq$ $1.5 \mathrm{kpc}$, seems highly implausible. Such a case would demand a very large Fornax dark halo: $V_{\max } \gtrsim 200 \mathrm{~km} \mathrm{~s}^{-1}$ with $M\left(<r_{\max }\right) \simeq 4.4 \times 10^{11} \mathrm{M}_{\odot}$. The dynamical friction timescale 
for such an object at a distance of $\sim 140 \mathrm{kpc}$ is less than $\sim 0.5 \mathrm{Gyr}$. Consider also the fact that the stellar mass of Fornax is $M_{\star} \simeq 3 \times 10^{7} \mathrm{M}_{\odot}$ (Mateo 1998). This means that a $\sim 1.5 \mathrm{kpc}$ core in Fornax would require both that the Milky Way is experiencing a (very rare) recent equal-mass merger and that the merging object is a system with a stellar baryon fraction that is $\sim 10,000$ times smaller than the universal baryon fraction.

\section{CONCLUSIONS AND DISCUSSION}

In this paper, we have shown that a standard WDM particle cannot produce a core in Fornax larger than $\sim 85 \mathrm{pc}$ without saturating the implied $\operatorname{Ly} \alpha$ forest bound on the phase-space density. For a more general class of dark matter, the relation between the phase-space density and the power spectrum may be different, and in these cases the limit on $Q$ comes from low-mass galaxy rotation curves, giving $r_{\text {core }} \lesssim 300$ pc. Irrespective of the origin of the core, dynamical arguments alone place an upper limit on the Fornax halo mass and demand $r_{\text {core }} \lesssim 700$ pc.

One important caveat to the above results is that there is no concrete prediction for the precise shape or normalization of dark matter halo profiles in cases where the dark matter is not cold. Even in well-studied cases like WDM, a phase-space core has never been realized in an N-body simulation and the nature of the expected transition between a rising $\rho(r)$ profile and the flat core region is unknown. The density profile we have adopted in equation 2 asymptotes to the CDM prediction at large radius, $\rho(r) \propto r^{-3}$ and allows some freedom in the nature of the transition from the inner core region to the outer fall-off in density. The sharpness of this transition is captured in the shape parameter $\alpha$ : the sharper the transition (larger $\alpha$ ), the closer the core radius is to the radius of maximum rotation. Given our general ignorance as to the "correct" shape, we have explored the degree to which our constraints on the core size change when we adopt $\alpha=1$ and 2 . The $Q$ constraint for a broad class of dark matter models derived from from low-mass spiral galaxies yields $r_{\text {core }} \lesssim 500 \mathrm{pc}$ and 200 pc for $\alpha=2$ and 1, respectively. For WDM, the Ly $\alpha$ forest power spectrum implies $r_{\text {core }} \lesssim 150 \mathrm{pc}$ and $40 \mathrm{pc}$, for $\alpha=2$ and 1 , respectively.

What do our results mean for the globular clusters in Fornax? A core large enough to explain the extended distribution of globular clusters, $r_{\text {core }} \gtrsim 1.5 \mathrm{kpc}$, would imply a dynamically implausible dark halo, $V_{\max } \gtrsim 200 \mathrm{~km} \mathrm{~s}^{-1}$ (see Figure 2). In addition, such a core is vastly inconsistent with any viable WDM model.

Given these constraints, an alternative explanation for the globular cluster system may be considered. For example, the profile could be cuspy, but this would require the globular clusters to have formed at large radii beyond the asymptotic central slope (J. Read, private communication). This seems to require a fine-tuned timing argument. The timing argument could be sightly alleviated by arguing that only the innermost globular cluster defines the core $\left(r_{\text {core }} \gtrsim 240 \mathrm{pc}\right)$, however even this case is inconsistent with standard WDM.

We may also consider a solution based on tidal heating. For example, dynamical friction effects on the orbital decay of the globular clusters can be counteracted by dynamical heating via tidal shocks or by relaxation during major mergers (Oh et al. 2000). The tidal heating explanation may have difficulty because the proper motion measurements indicate that the Fornax is currently near its orbital pericenter and has apocenter of $\approx 250 \mathrm{kpc}$ (Dinescu et al. 2004) and thus does not come sufficiently close to the center of the Milky Way to experience strong tidal force. Note, however, that the tidal heating could have been provided by another object in the past and not necessarily by the Milky Way progenitor (see, e.g., Kravtsov et al. 2004). Heating by a major merger with another dwarf galaxy is plausible, as there is evidence for such recent major merger in Fornax from observations of extra-tidal stars (Coleman et al. 2005; Olszewski et al. 2006). Although such mergers are not likely for satellites orbiting in a potential of a much larger host halo, given large pericentric and apocentric distances of the Fornax, it is likely that it has been accreted by the Milky Way only recently. Major mergers are much more likely prior to accretion by a large host. Other possible, albeit more speculative, explanations, such as core formation due to inspiralling of multiple black holes, are discussed in SanchezSalcedo et al. (2006).

As emphasized by Goerdt et al. (2006) and SanchezSalcedo et al. (2006), the Fornax globular cluster system presents an interesting case study with possible fundamental implications for the nature of dark matter. Here we argue that interpretation in terms of large dark matter core is problematic, as such a large core is not consistent with popular dark matter alternatives to CDM. Large cores also appear to be inconsistent with high-resolution measurements of rotation curves of the LSB galaxies for an even broader class of dark matter scenarios. Dynamical heating of the globular clusters by tides or relaxation in mergers could provide an alternative explanation and needs to be explored.

We are grateful to Terry Walker and the Center for Cosmology and Astro-Particle Physics (CCAPP) at The Ohio State University for hosting the workshop on alternative dark matter models in January of 2006, where this work was initiated. We thank Ben Moore, Justin Read, and Andrew Zentner for enlightening discussions. LES is supported in part by a Gary McCue Postdoctroral Fellowship through the Center for Cosmology at UC Irvine. AVK is supported by the NSF grants AST-0206216, AST-0239759, and AST-0507596, by NASA through grant NAG5-13274, and by the Kavli Institute for Cosmological Physics at the University of Chicago. KA is supported by Los Alamos National Laboratory under DOE contract W-7405-ENG-36. AK is supported by NSF grant AST-0407072. We acknowledge Larry's for inspiration.

\section{REFERENCES}

Abazajian, K. 2006a, Phys. Rev. D, 73, 063506

-. 2006b, Phys. Rev. D, 73, 063513

Abazajian, K., Fuller, G. M., \& Patel, M. 2001, Phys. Rev. D, 64, 023501

Alam, S. M. K., Bullock, J. S., \& Weinberg, D. H. 2002, ApJ, 572, 34

Blumenthal, G. R., Pagels, H., \& Primack, J. R. 1982, Nature, 299, 37

Bond, J. R., Efstathiou, G., \& Silk, J. 1980, Physical Review Letters, 45, 1980

Buonanno, R., Corsi, C. E., Castellani, M., Marconi, G., Fusi Pecci, F., \&

Zinn, R. 1999, AJ, 118, 1671

Burkert, A. 1995, ApJ, 447, L25+
Cembranos, J. A., Feng, J. L., Rajaraman, A., \& Takayama, F. 2005, PRL, 95, 181301

Cole, S. \& Lacey, C. 1996, MNRAS, 281, 716

Coleman, M. G., Da Costa, G. S., Bland-Hawthorn, J., \& Freeman, K. C. 2005, AJ, 129, 1443

Colín, P., Klypin, A. A., \& Kravtsov, A. V. 2000, ApJ, 539, 561

Dalcanton, J. J. \& Hogan, C. J. 2001, ApJ, 561, 35

de Blok, W. J. G., McGaugh, S. S., Bosma, A., \& Rubin, V. C. 2001, ApJ, 552, L23 
Diemand, J., Moore, B., \& Stadel, J. 2004, MNRAS, 352, 535

Diemand, J., Zemp, M., Moore, B., Stadel, J., \& Carollo, M. 2005, MNRAS 364,665

Dinescu, D. I., Keeney, B. A., Majewski, S. R., \& Girard, T. M. 2004, AJ 128,687

Dodelson, S. \& Widrow, L. M. 1994, Physical Review Letters, 72, 17

Dubinski, J. \& Carlberg, R. G. 1991, ApJ, 378, 496

Faltenbacher, A., Kravtsov, A. V., Nagai, D., \& Gottlöber, S. 2005, MNRAS, 358,139

Flores, R. A. \& Primack, J. R. 1994, ApJ, 427, L1

Goerdt, T., Moore, B., Read, J. I., Stadel, J., \& Zemp, M. 2006, MNRAS submitted (astro-ph/0601404), G06

Hogan, C. J. \& Dalcanton, J. J. 2000, Phys. Rev. D, 62, 063511

Hu, W., Barkana, R., \& Gruzinov, A. 2000, Physical Review Letters, 85, 1158

Kaplinghat, M. 2005, Phys. Rev. D, 72, 063510

Kaplinghat, M., Knox, L., \& Turner, M. S. 2000, Physical Review Letters, 85,3335

Kazantzidis, S., Mayer, L., Mastropietro, C., Diemand, J., Stadel, J., \& Moore, B. 2004, ApJ, 608, 663

Klypin, A., Kravtsov, A. V., Bullock, J. S., \& Primack, J. R. 2001, ApJ, 554, 903

Klypin, A., Kravtsov, A. V., Valenzuela, O., \& Prada, F. 1999, ApJ, 522, 82

Kormendy, J. \& Freeman, K. C. 2004, in IAU Symposium, 377-+

Kravtsov, A. V., Gnedin, O. Y., \& Klypin, A. A. 2004, ApJ, 609, 482

Kuhlen, M., Strigari, L. E., Zentner, A. R., Bullock, J. S., \& Primack, J. R. 2005, MNRAS, 357, 387

Łokas, E. L. 2002, MNRAS, 333, 697

Mackey, A. D. \& Gilmore, G. F. 2003, MNRAS, 340, 175

Mateo, M. L. 1998, ARA\&A, 36, 435

Mayer, L. \& Wadsley, J. 2004, in ASP Conf. Ser. 327: Satellites and Tidal Streams, 230-+

Medvedev, M. V. 2000, preprint (astro-ph/0010616)

Moore, B. 1994, Nature, 370, 629

Moore, B., Ghigna, S., Governato, F., Lake, G., Quinn, T., Stadel, J., \& Tozzi, P. 1999, ApJ, 524, L19
Navarro, J. F., Frenk, C. S., \& White, S. D. M. 1996, ApJ, 462, 563

Navarro, J. F., Hayashi, E., Power, C., Jenkins, A. R., Frenk, C. S., White, S. D. M., Springel, V., Stadel, J., \& Quinn, T. R. 2004, MNRAS, 349, 1039 Oh, K. S., Lin, D. N. C., \& Richer, H. B. 2000, ApJ, 531, 727

Olszewski, E. W., Mateo, M., Harris, J., Walker, M. G., Coleman, M. G., \& Da Costa, G. S. 2006, AJ, 131, 912

Pagels, H. \& Primack, J. R. 1982, Physical Review Letters, 48, 223

Pont, F., Zinn, R., Gallart, C., Hardy, E., \& Winnick, R. 2004, AJ, 127, 840

Prada, F., Vitvitska, M., Klypin, A., Holtzman, J. A., Schlegel, D. J., Grebel, E. K., Rix, H.-W., Brinkmann, J., McKay, T. A., \& Csabai, I. 2003, ApJ, 598,260

Sánchez-Salcedo, F. J. 2003, ApJ, 591, L107

Sanchez-Salcedo, F. J., Reyes-Iturbide, J., \& Hernandez, X. 2006, MNRAS submitted (astro-ph/0601490)

Seljak, U., Makarov, A., McDonald, P., \& Trac, H. 2006, Phys. Rev. Lett., submitted (astro-ph/0602430)

Sellwood, J. A. 2000, ApJ, 540, L1

Shi, X. \& Fuller, G. M. 1999, Physical Review Letters, 82, 2832

Simon, J. D., Bolatto, A. D., Leroy, A., Blitz, L., \& Gates, E. L. 2005, ApJ, 621,757

Spergel, D. N. \& Steinhardt, P. J. 2000, Physical Review Letters, 84, 3760

Taylor, J. E. \& Navarro, J. F. 2001, ApJ, 563, 483

Tremaine, S. \& Gunn, J. E. 1979, Physical Review Letters, 42, 407

Viel, M., Lesgourgues, J., Haehnelt, M. G., Matarrese, S., \& Riotto, A. 2005, Phys. Rev. D, 71, 063534

Walker, M. G., Mateo, M., Olszewski, E. W., Bernstein, R. A., Wang, X., \& Woodroofe, M. 2005, AJ in press (astro-ph/0511465)

Wojtak, R., Łokas, E. L., Gottlöber, S., \& Mamon, G. A. 2005, MNRAS, 361, L1

Zackrisson, E., Bergvall, N., Marquart, T., \& Ostlin, G. 2006, preprint (astro$\mathrm{ph} / 0603523)$

Zentner, A. R. \& Bullock, J. S. 2002, Phys. Rev. D, 66, 043003

-. 2003, ApJ, 598, 49 